(C) 2007 The Japan Society of Applied Physics

\title{
Lipid-Modulated Assembly of Magnetized Iron-Filled Carbon Nanotubes in Millimeter-Scale Structures
}

\author{
Nashville C. Toledo, Maurits R. R. De Planque*, Sonia Antoranz Contera, \\ Nicole GROBERT ${ }^{1}$, and John F. RYAN \\ Bionanotechnology IRC, Clarendon Laboratory, Department of Physics, University of Oxford, \\ Parks Road, Oxford OX1 3PU, United Kingdom \\ ${ }^{1}$ Department of Materials, University of Oxford, Parks Road, Oxford OX1 3PH, United Kingdom
}

(Received September 19, 2006; accepted November 21, 2006; published online April 24, 2007)

Biomolecule-functionalized carbon nanotubes (CNTs) combine the molecular recognition properties of biomaterials with the electrical properties of nanoscale solid state transducers. Application of this hybrid material in bioelectronic devices requires the development of methods for the reproducible self-assembly of CNTs into higher-order structures in an aqueous environment. To this end, we have studied pattern formation of lipid-coated Fe-filled CNTs, with lengths in the 1-5 $\mu \mathrm{m}$ range, by controlled evaporation of aqueous CNT-lipid suspensions. Novel diffusion limited aggregation structures composed of endto-end oriented nanotubes were observed by optical and atomic force microscopy. Significantly, the lateral dimension of assemblies of magnetized Fe-filled CNTs was in the millimeter range. Control experiments in the absence of lipids and without magnetization indicated that the formation of these long linear nanotube patterns is driven by a subtle interplay between radial flow forces in the evaporating droplet, lipid-modulated van der Waals forces, and magnetic dipole-dipole interactions. [DOI: 10.1143/JJAP.46.2799]

KEYWORDS: nano-structures, carbon nanotubes, lipid bilayers, diffusion limited aggregation, self-assembly, bioelectronics

\section{Introduction}

An increasing research effort is directed at combining the electrical properties of solid state materials with the specific recognition and catalytic properties of biomaterials such as proteins and DNA, in order to create bioelectronic systems with novel functionalities. ${ }^{1)}$ As an example, electronic biosensors could be designed in which the analyte of interest binds specifically to a protein that is in electronic communication with a transducing substrate, giving rise to an electrical signal that marks the molecular binding event and thereby the presence of the analyte. The biochemically relevant size range is $1 \mathrm{~nm}$ to $1 \mu \mathrm{m}$, enabling the presence of a large number of biosensors (with specificity for different compounds) on a chip-sized surface. However, the integration of biomolecules with electronic elements is not trivial, primarily because biomolecules have not evolved to interact with solid substrates and only function in an electrolytic aqueous solution.

The cells of all living organisms are enclosed by a cell membrane that consists of a bilayer of amphipathic lipid molecules with embedded proteins. Simplified versions of these membranes are attractive components for bioelectronic elements because lipid bilayers are electrically insulating whereas proteins can be introduced to allow for transmembrane communication. The ion channel receptor proteins that are abundant components of cell membranes are highly selective and transmit extracellular binding events to the interior of the cell by temporarily opening their internal transmembrane channel, enabling an electrolyte flow that, for an individual channel protein, gives rise to a current in the $\mathrm{pA}-\mathrm{nA}$ range at applied potentials in the $\mathrm{mV}$ range. ${ }^{2)}$ Moreover, affinity of the membrane proteins themselves for the transducing solid substrate would not be required if the interaction of the membrane surface with the

*E-mail address: m.deplanque1@physics.ox.ac.uk substrate is determined by the lipid components of the membrane.

Carbon nanotubes (CNTs), cylinders of graphene sheets with a diameter of $1-200 \mathrm{~nm}$ and a length of $1-100 \mu \mathrm{m}$, are attractive solid components for bioelectronic devices because of their small size, high aspect ratio, significant strength, and high thermal and electrical conductivity.,4) CNTs have been conjugated with a variety of biomolecules, including DNA, peptides, and soluble proteins, ${ }^{3-5)}$ but not with biological membranes. However, protein-free model membranes consisting of a self-assembling bilayer of synthetic lipid molecules can be formed on planar solid substrates by surface-induced rupture of small vesicular lipid assemblies. ${ }^{6,7)}$ Solid-supported bilayers of commercially available synthetic lipids have a thickness of $4-5 \mathrm{~nm}$, a capacitance of $\sim 0.5 \mu \mathrm{F} / \mathrm{cm}^{2}$ and a resistance of $\geq 10$ $\mathrm{M} \Omega \cdot \mathrm{cm}^{2}$, and thus electrically insulate the transducing substrate from the electrolyte aqueous solution despite their non-biological composition. ${ }^{8)}$

The potential of membrane-based electronic biosensors has been demonstrated by the development and commercialization of an electrode-supported bilayer containing the small ion channel gramicidin. ${ }^{9)}$ The use of CNTs as the transducing solid support would enable further miniaturization of bioelectronic devices, but the nanotubes will have to be present as extended networks that can be connected to conventional electrical circuitry. Unfortunately, self-association of CNTs into well-defined higher-order structures presents a technological hurdle. ${ }^{3)}$ Electric field assisted alignment of CNTs on electrodes has been achieved in various organic solvents. ${ }^{10-12)}$ More recently, higher-order nanotube structures have been obtained by flow-directed evaporation of aqueous CNT suspensions, in particular droplet evaporation. When the perimeter of an evaporating droplet becomes pinned to the surface, further evaporation results in height but not in area reduction of the droplet, and the resulting radial capillary flow within droplets of 
concentrated CNT suspensions has been shown to induce side-to-side alignment of nanotubes. ${ }^{13-17)}$

In this paper, we compare the patterns that are formed by evaporation of droplets of dilute aqueous suspensions of uncoated iron-filled multi-walled CNTs (Fe-filled MWNTs) with the patterns that are formed by Fe-filled MWNTs that are coated with lipid bilayers. Fe-filled MWNTs were employed because their relatively large diameter is favorable for lipid bilayer attachment while the iron core renders these CNTs ferromagnetic, enabling manipulation by external magnetic fields. Symmetric fractals and millimeter-length linear assemblies of end-to-end oriented nanotubes were obtained. These patterns were only formed in the presence of lipids and with Fe-filled MWNTs that had been magnetized prior to droplet evaporation, highlighting the complex interplay of forces that govern CNT self-assembly. Such large assemblies of end-to-end, rather than side-to-side, oriented lipid-coated CNTs appear promising components for electronic biosensors.

\section{Materials and Methods}

\subsection{Materials}

Fe-filled MWNTs were grown by pyrolizing mixtures of powdered ferrocene and $\mathrm{C}_{60}$ at $900-1050{ }^{\circ} \mathrm{C}$ in an argon flow using a conventional two-stage furnace. As-grown $\mathrm{Fe}-$ filled MWNTs have external diameters of $\sim 20-70 \mathrm{~nm}$, are $<40 \mu \mathrm{m}$ in length and exhibit a magnetic coercivity of $\sim 450$ Oe at room temperature. ${ }^{18)}$ This material was sonicated in a mixture of concentrated sulfuric and nitric acid to introduce carboxylic groups on the nanotube surface, which prevent immediate aggregation of CNTs in aqueous solution. This acid treatment also reduces the nanotube length to $\sim 1 \mu \mathrm{m}$. The acidic Fe-filled MWNT suspension was filtered to collect the nanotubes, which were subsequently washed on the filter with distilled water until the acidity of the filtrate was reduced to $\mathrm{pH}$ 6. Alternatively, the nanotubes were collected from the concentrated acid mixture by placing a stack of magnets next to the vial, followed by repeated resuspension in distilled water, again with collection of the Fe-filled MWNTs by magnetic attraction, until a $\mathrm{pH}$ value of 6 was measured. The synthetic lipid 1,2-dioleoyl-sn-glycero-3-phosphatidylcholine (DOPC) was obtained from Avanti Polar Lipids, and neodymium-iron-boron block magnets (dimensions $20 \times$ $10 \times 5 \mathrm{~mm}^{3}$, magnetization directed from the top to the bottom $20 \times 10 \mathrm{~mm}^{2}$ face, remanence $B_{\mathrm{r}} \sim 1100 \mathrm{mT}$ ) from IBS Magnets.

\subsection{Sample preparation}

For lipid-containing samples, a dispersion of sonicated lipid vesicles was added to an aqueous CNT suspension in distilled water $(\sim 30 \mu \mathrm{g} / \mathrm{mL})$. After an overnight incubation period the majority of vesicles that had not associated with the nanotubes were separated from the nanotubes by a single centrifugation step. The same procedure, but without adding lipid vesicles, was followed for control samples of acidtreated Fe-filled MWNTs. The pelleted nanotubes were subsequently resuspended in distilled water $(\sim 60 \mu \mathrm{g} / \mathrm{mL})$, and droplets with a volume of $5 \mu \mathrm{L}$ were allowed to slowly evaporate on a glass slide (J. Melvin Freed Brand) that was placed inside a polystyrene petri dish $(90 \mathrm{~mm}$ diameter,

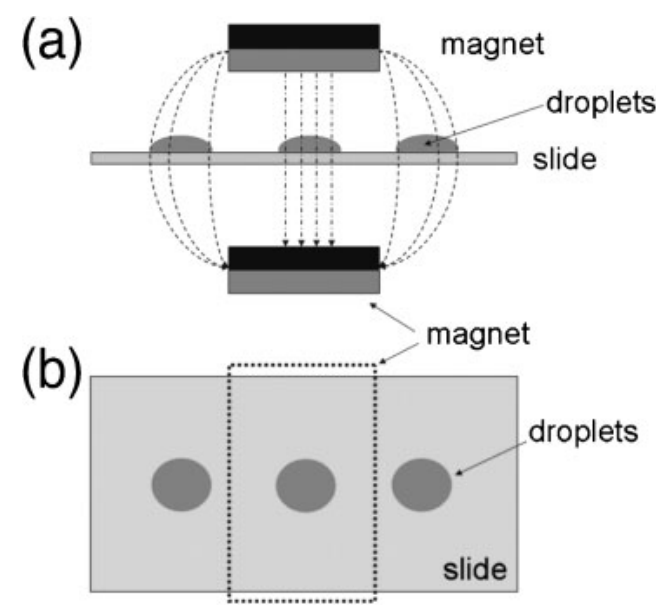

Fig. 1. Schematic side view (a) and top view (b) of the experimental setup used for evaporation of droplets of CNT suspensions in an external magnetic field. The two block magnets are positioned in attraction, $20 \mathrm{~mm}$ apart and with a glass slide (inside a petri dish; not shown) between them. Droplets were placed on the slide, directly under and on either side of the magnets. Fe-filled MWNTs in the droplet at the central position experience a relatively homogenous vertical field while nanotubes in the off-center droplets will experience fringe fields.

triple vent). The evaporation process typically took $4 \mathrm{~h}$ to complete. For droplet evaporation under the influence of an external magnetic field, the glass slide, again inside a petri dish, was positioned between two block magnets as depicted in Fig. 1.

\subsection{Optical and atomic force microscopy}

The nanotube patterns that were created by complete evaporation of droplets on a glass slide were examined with a Nikon TE-2000 microscope with a $10 \times$ Plan Fluor objective, and reflected light differential interference contrast (DIC) images were captured with a Hamamatsu C848405G digital CCD camera. For atomic force microscopy (AFM) of selected areas of the nanotube patterns, the same glass slides were mounted on the scanner of a stand-alone Asylum MFP-3D AFM, with a closed loop in the $x, y$, and $z$ axes. Experiments were performed in AC-mode with Olympus AC240TS cantilevers with a nominal spring constant of $1.8 \mathrm{~N} / \mathrm{m}$.

\section{Results and Discussion}

\subsection{Droplet drying of Fe-filled MWNT suspensions}

DIC phase microscopy images of evaporated droplets of centrifugated CNT suspensions, with and without lipids, are shown in Fig. 2. In the absence of an external magnetic field, acid-treated Fe-filled MWNTs that have not been in contact with lipids (uncoated nanotubes), became deposited as amorphous aggregates with apparent cross-sections of $\leq 10 \mu \mathrm{m}$ [Fig. 2(a)]. The even distribution of these aggregates and the lack of larger aggregates illustrate that acid treatment significantly improves the stability of aqueous CNT suspensions. The aggregates were relatively uniformly spread out over the circular area covered by the evaporated droplet, with only a slightly higher concentration at the perimeter (not shown).

In contrast, the evaporation of a suspension of Fe-filled MWNTs that have been incubated with lipid vesicles (coated 

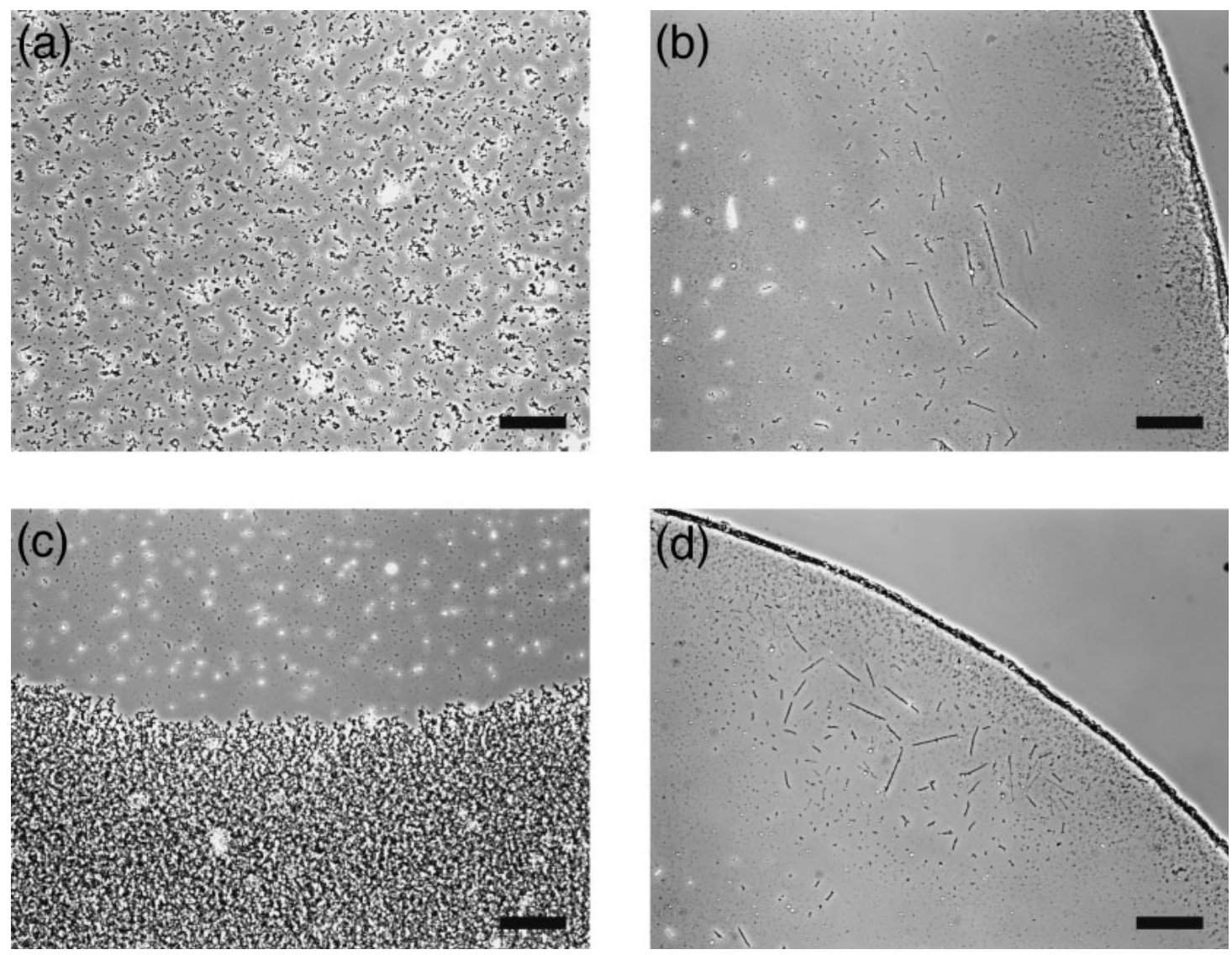

Fig. 2. Optical microscopy image of the evaporation pattern of droplets of uncoated (a,c) and lipid-coated (b,d) Fe-filled MWNTs: (a) and (b) after evaporation in the absence of an external magnetic field, (c) and (d) after evaporation at off-center positions with respect to the external magnetic field. Scale bars are $100 \mu \mathrm{m}$.

nanotubes) gave rise to the markedly different deposition pattern that is shown in Fig. 2(b). Linear assemblies of CNTs, with an apparent cross-section of $\sim 2 \mu \mathrm{m}$ and a length that ranges from approximately 10 to $100 \mu \mathrm{m}$, were present at a distance of $\sim 400 \mu \mathrm{m}$ from the droplet edge. This edge is visible at the right side of the figure as a dense boundary with a cross-section of about $20 \mu \mathrm{m}$. The center of the evaporated droplet was essentially devoid of nanotubes while the edge was clearly enriched with the smallest nanotube assemblies that can be observed at the employed magnification. The absence of amorphous aggregates demonstrates that the CNTs have effectively been solubilized by the lipids, and complete lipid bilayer coating of the nanotubes was confirmed by fluorescence microscopy and AFM. ${ }^{19)}$

The formation of a pronounced perimeter of deposited solute results from the constraints that are imposed upon the evaporation process when the contact line of a drying droplet becomes pinned to the substrate. ${ }^{13,20)}$ Evaporative losses at the contact line are compensated by an outward capillary flow (from the center of the droplet) that carries almost the entire amount of solubilized or suspended material toward the pinned edge, provided that the hydrodynamic drag on the solute dominates forces that exert a pull in other directions. This is not the case for the aggregates of uncoated nanotubes, the high density of which has led to gravityinduced deposition on a large surface area [Fig. 2(a)]. Also the lipid-coated nanotubes have not entirely been transported to the droplet edge, but their assembly state depends on the distance from the edge, with the linear assemblies typically further removed from the perimeter than the much smaller dot-shaped material [Fig. 2(b)]. This belt of linear CNT assemblies may mark the radial point on the surface of an evaporating droplet where the drag of the surface tension gradient (toward the center of the drop) balances the capillary flow of the underlying liquid (toward the edge). ${ }^{20)}$ It is not clear at what stage of the evaporation process the organization of coated nanotubes into well-separated lines is induced, but such patterns are relevant for the development of self-assembling (nano)electrical circuitry. ${ }^{1)}$

To investigate the influence of an external magnetic field on pattern formation, the suspensions of uncoated and coated Fe-filled MWNTs were also allowed to evaporate at various positions between two permanent block magnets, as schematically depicted in Fig. 1. The ferromagnetic nanotubes had not been magnetized during the sample preparation process. Evaporation of the off-center droplets of uncoated CNT suspension resulted in the accumulation of amorphous aggregates on the side of the drop that points away from the center position of the magnetic field [Fig. 2(c)]. This accumulation was also observed when the magnets were positioned to the left and to the right of the sample (not illustrated), but when the same suspension was dried out at the center position with the magnets above and below the sample (Fig. 1), the aggregates deposited in a uniform manner over the entire surface of the droplet footprint (data 
not shown). These observations demonstrate that with the magnet configuration shown in Fig. 1, the magnetic field is sufficiently non-linear at the off-center droplet positions to cause lateral displacement of susceptible solutes.

A clear influence of the magnetic field on the deposition pattern of lipid-coated Fe-filled MWNTs was, however, not observed. The linear assemblies of nanotubes that are visible in Fig. 2(d) are not longer and do not exhibit a clearly altered orientation or intertube separation than the coated nanotubes that were deposited in the absence of a magnetic field [Fig. 2(b)], although they do lie closer to the edge of the evaporated droplet. The absence of a more pronounced effect of the external magnetic field may be due to the effective solubilization of the CNTs by the lipid; a stronger external force will be required to disrupt the displacing effect of the capillary flow of the evaporating droplet for small nanotube assemblies than for high-density aggregates. ${ }^{13,20)}$ Another consideration is that the residual lipid vesicles that are present in the suspension will also deposit during the drying process, resulting in the formation of planar lipid bilayers on the glass substrate. ${ }^{6,7)}$ This lipid deposition will be modulated by the capillary flow but not by the magnetic field, and if the coated nanotubes display a specific affinity for the planar lipid bilayers, the magnetic force may not be of sufficient strength to displace the Fefilled MWNTs from these lipid domains.

\subsection{Droplet drying of pre-magnetized Fe-filled MWNT suspensions}

As described in $\$ 2$, the Fe-filled MWNTs were also processed with prolonged exposure $(\sim 48 \mathrm{~h})$ to an external magnetic field, leading to magnetization of these ferromagnetic nanotubes prior to droplet evaporation. However, it should be noted that this "pre-magnetization" step is not expected to impart the entire nanotube population with a well-defined magnetic dipole moment, because not all the tubes are completely filled with $\mathrm{Fe}$, and not all the iron atoms are present in the ferromagnetic $\alpha$-Fe configuration. ${ }^{18,21-25)}$ Droplet drying of pre-magnetized uncoated nanotubes in the absence and presence of an external magnetic field (data not shown) gave rise to similar deposition patterns as those shown in Figs. 2(a) and 2(c) for non-magnetized nanotubes, suggesting that inter-tube dipolar interactions are dominated by the (van der Waals) forces that drive aggregation of uncoated CNTs. Indeed, in related studies on spherical paramagnetic particles, pattern formation could only be observed when the hydrophobic particle surface was functionalized with negatively charged detergents such as sodium dodecyl sulfate. ${ }^{26-29)}$ This procedure introduces electrostatic inter-particle repulsion to counteract van der Waals attraction, thereby enabling magnetic interactions to modulate higher order particle organization.

Lipid coating of CNTs abolishes the dominant role of van der Waals interactions, but does not lead to pronounced electrostatic inter-tube repulsion because the head group of the employed DOPC lipid is zwitterionic. ${ }^{30,31)}$ In fully hydrated stacks of planar DOPC bilayers, the water layer which separates two bilayers is only $2.5 \mathrm{~nm} .{ }^{30-32)}$ The shortrange repulsive forces which balance the attractive van der Waals force at spacings of $1-3 \mathrm{~nm}$ are due to a
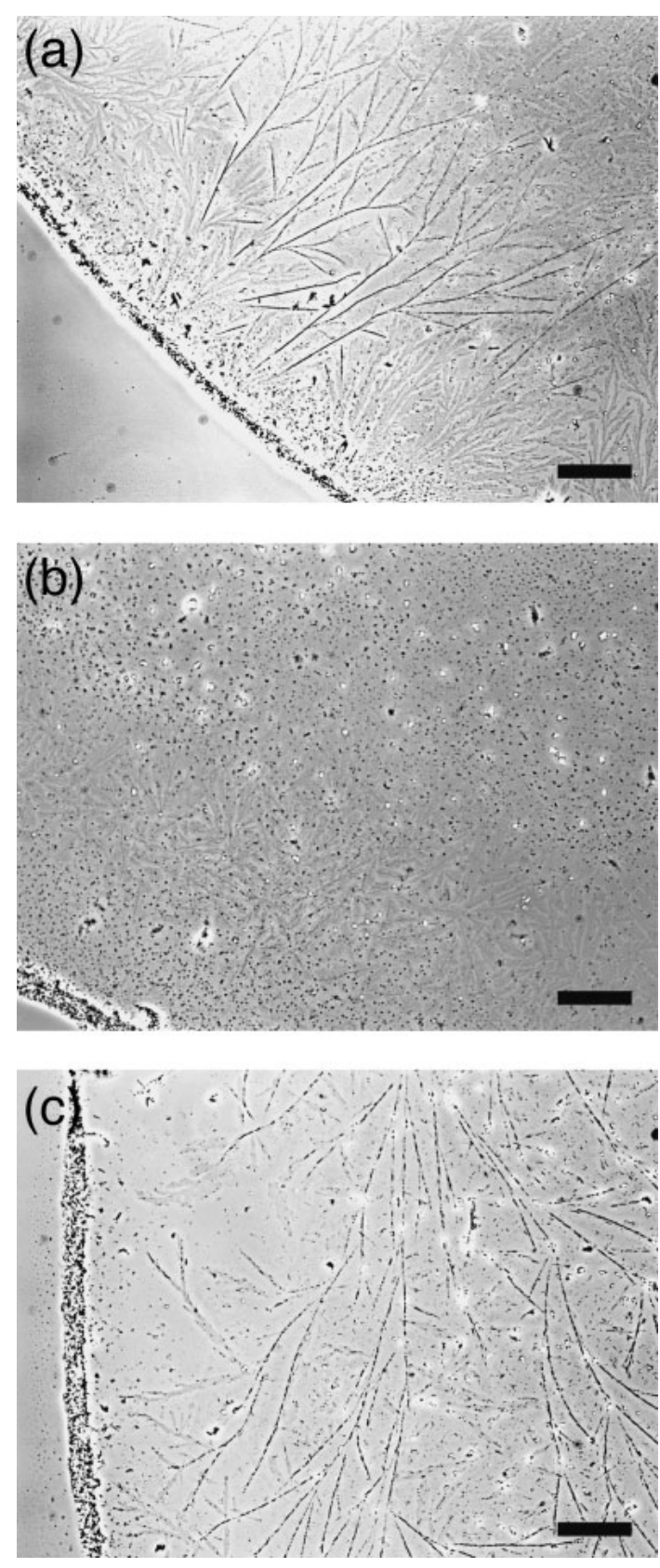

Fig. 3. Optical microscopy image of the evaporation pattern of droplets of lipid-coated Fe-filled MWNTs that have been magnetized prior to droplet placement: (a) after evaporation in the absence of an external magnetic field, (b) after evaporation at the central position, and (c) at an off-center position with respect to the external magnetic field. Nanotubes appear as black features and lipid vesicle deposits as light grey patterns. Scale bars are $100 \mu \mathrm{m}$.

combination of hydration and steric repulsions, the latter arising from thermal motions of lipid head groups and thickness fluctuations of lipid bilayers. ${ }^{30,31)}$ The dipoledipole interactions between pre-magnetized lipid-coated Fefilled MWNTs may therefore be of sufficient strength, relative to the combined effect of other forces, to modulate the manner in which these nanotubes deposit from a drying droplet. A representative evaporation pattern for the corresponding CNT suspension in the absence of an external magnetic field, shown in Fig. 3(a), is indeed markedly different from the pattern formed by coated tubes that had not been explicitly magnetized [Fig. 2(b)]. Unlike the latter 
CNTs, the pre-magnetized coated nanotubes had assembled in a large number of relatively straight linear features, with lengths of up to $\sim 300 \mu \mathrm{m}$, all oriented toward the edge of the evaporated droplet and exhibiting an increased extent of branching further away from the edge.

The increased tendency of the pre-magnetized coated nanotubes to self-associate in a linear, presumably end-toend, fashion, is a typical manifestation of dipolar coupling. ${ }^{28,29,33)}$ However, in the absence of an external magnetic field, dipole-dipole interactions are not expected to lead ferromagnetic particles to cluster as relatively straight lines but rather as randomly oriented branched chains. ${ }^{29)}$ Such clusters can resemble self-similar aggregates with a relatively large fractal dimension, particularly in the case of small particles that experience relatively weak dipolar coupling, ${ }^{29,33)}$ as is the case for the pre-magnetized Fe-filled nanotubes. The observation that these tubes have assembled as relatively straight lines with a low degree of branching and a radial orientation, particularly close to the edge of the evaporation pattern [Fig. 3(a)], can thus be attributed to the capillary flow toward the droplet edge. As the distance from the edge increases, the hydrodynamic drag on the nanotubes weakens and the dipole-dipole interactions become able to induce branching, particularly in the stagnation belt.

When the droplet with pre-magnetized coated nanotubes was evaporated at the central position of an external perpendicular magnetic field, linear CNT structures were not formed. As can be seen in Fig. 3(b), a large amount of nanotubes was homogeneously dispersed over the interior of the droplet footprint, apparently 'pinned down' by the perpendicular magnetic field. The pre-magnetized tubes are thus sensitive to the applied magnetic field, but the external magnetic forces are not strong enough to entirely prevent flow-induced effects on nanotube deposition, as follows from the existence of a nanotube-rich droplet edge and also from the depletion of CNTs near this edge.

For droplets that were evaporated at an off-center position in the perpendicular magnetic field, linear nanotube assemblies were again observed [Fig. 3(c)]. Significantly, the dark nanotube lines (light grey features are lipid domains; see below) had lengths in the millimeter range and were well separated from each other: side-to-side association of CNTs did not occur. A long central line had formed in the stagnation belt, running parallel to the edge of the droplet at a separation distance of about $400 \mu \mathrm{m}$, with shorter nanotube lines branching out in opposite directions from this central line. The nanotube branches pointing toward the edge of the droplet appear directed by the capillary flow while those pointing to its center by the surface tension gradient. The CNT patterns, which resemble two-dimensional diffusion limited aggregation (DLA) structures, ${ }^{34)}$ thus cover a larger area than in the absence of a magnetic field. These observations suggest that in the employed experimental setup, the radial flow forces are counteracted by the external magnetic force over a relatively large area of the evaporating droplet, enabling diffusion limited processes to modulate CNT assembly.

\subsection{Assembly of Fe-filled MWNT structures}

To verify the composition of the linear structures a

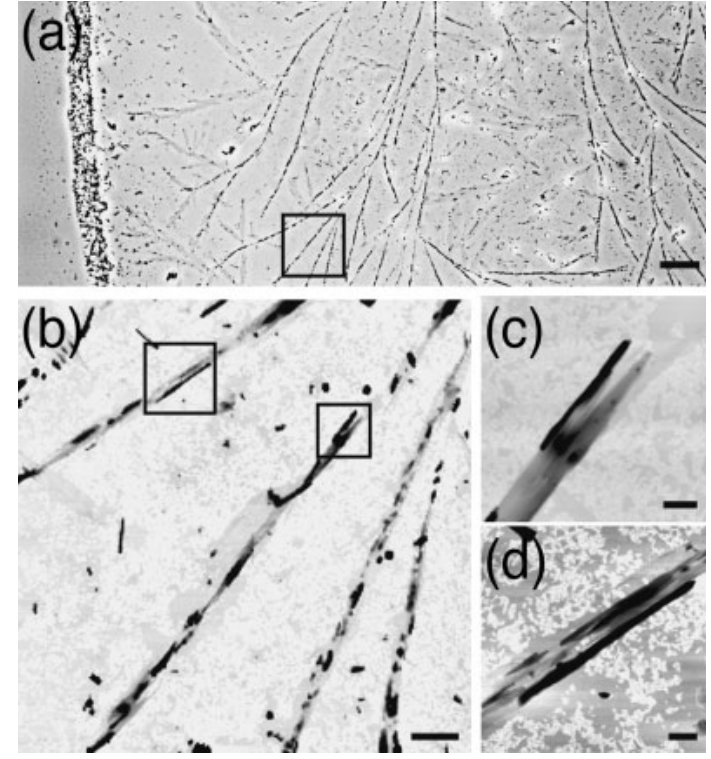

Fig. 4. Optical microscopy (a) and AFM (b-d) images of linear assemblies of lipid-coated pre-magnetized Fe-filled MWNTs, obtained after evaporation of the CNT suspension at an off-center position in an external magnetic field. (b) AC-AFM image of the boxed region in (a): the glass is white, lipid bilayers patches are grey, higher features are black. (c,d) AFM images of the boxed regions in (b): the glass is white, lipid bilayer patches are light grey, lipid multilayer patches are dark grey, CNTs are black. The z-range is $100 \mathrm{~nm}$ in (b) and (c) and $50 \mathrm{~nm}$ in (d) Scale bars are $50 \mu \mathrm{m}$ in (a), $5 \mu \mathrm{m}$ in (b), and $1 \mu \mathrm{m}$ in (c) and (d).

selected area of the sample shown in Fig. 3(c) was investigated by AFM. The black features in the optical microscopy images were assigned to carbon nanotubes because DIC microscopy is sensitive to refractive index inhomogeneities and emphasizes height transitions. The AFM images in Figs. 4(b)-4(d) confirm the presence of carbon nanotubes, which can be recognized by their height and high aspect ratio, in the linear features discussed above. The nanotubes in the investigated part of the sample occur in a length range of $0.5-5 \mu \mathrm{m}$ and have a diameter of $<100$ $\mathrm{nm}$. The lines themselves have a width of $\sim 1 \mu \mathrm{m}$ and do not consist entirely of CNTs. The height of $\sim 20-40 \mathrm{~nm}$ and the flatness of the material that is present between the nanotubes is typical for lipid multilayers. The area between two different nanotube lines is relatively empty, except for an occasional isolated nanotube [Fig. 4(b)]. Flat patches with a height of $\sim 5 \mathrm{~nm}$, or a multiple of this value, are also observed; these lipid bilayer and multilayer domains result from the deposition of excess lipid vesicles that were not removed by the centrifugation process.

The linear assemblies that are visible with DIC microscopy are thus composed of nanotubes but also contain a large amount of lipids. It is not known at which stage of the evaporation process the formation of nanotube patterns occurs, but the radial position of these two-dimensional DLA-like structures indicates modulation by the surface tension gradient (see below), and this implies CNT assembly on the surface of the droplet, prior to complete evaporation. In this scenario, the residual lipid vesicles preferentially adhere to and rupture on the CNT patterns because they present obstacles to vesicle diffusion, especially during the last stages of droplet evaporation. An alternative interpre- 


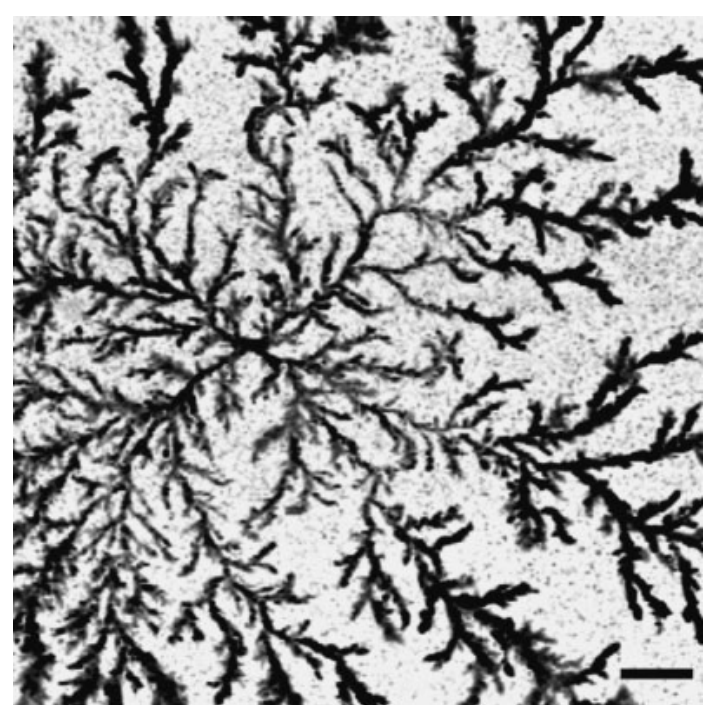

Fig. 5. AC-AFM image of a diffusion limited aggregation (DLA) structure of lipid-coated pre-magnetized Fe-filled MWNTs, obtained after evaporation of the CNT suspension in a magnetic field. The z-range is $100 \mathrm{~nm}$ and the scale bar is $2 \mu \mathrm{m}$.

tation is that the nanotube lines represent the last areas of fluid flux shortly before complete evaporation, when there is not enough water left to cover the entire droplet area. In this case, highly concentrated solutes, nanotubes as well as vesicles, would become deposited within the "rivers" of remaining suspension. This process would imply the formation of lipid bilayer trails, over which lipid-coated nanotubes may preferentially migrate until they become adsorbed to the surface or until the last water on the trail evaporates.

Pre-magnetized lipid-coated Fe-filled MWNTs that are deposited in a magnetic field, can also form symmetric clusters, rather than the asymmetric patterns described above, although these are only occasionally observed for a subpopulation of nanotubes with lengths of $200 \mathrm{~nm}$ or less. An expanded AFM image of such a two-dimensional structure, with an overall diameter of approximately $30 \mu \mathrm{m}$, is shown in Fig. 5. The fractal dimension of this two-dimensional CNT assembly as determined by boxcounting is 1.7. This value is typical for two-dimensional DLA clusters which grow by the addition of particles that are only subject to thermal diffusion before they become irreversibly attached to the cluster. ${ }^{34)}$ The occurrence of different types of DLA structures illustrates the complex nature of CNT pattern formation in our system.

The self-association of the magnetized nanotubes is driven by dipole-dipole interactions but the morphology and orientation of the CNT clusters are modulated by the radial flow toward the edge of the evaporating droplet, the surface tension gradient toward the center of the droplet, and the external magnetic field. Diffusion limited aggregation structures form around the radial position where the hydrodynamic forces cancel each other, ${ }^{20)}$ whereas outside this stagnation belt the radial flow leads to linear CNT assemblies with limited branching and a radial orientation [Fig. 3(a)]. The off-center magnetic field introduces an additional modulation of the diffusion properties of the nanotubes, resulting in the formation of extensive DLA clusters which branch out from the stagnation belt [Fig. 3(c)]. This morphology is expected to be sensitive to the magnitude and orientation of the magnetic field. The self-assembly of higher-order nanotube structures only occurs when the nanotubes are coated with lipids; uncoated CNTs consistently form amorphous aggregates. The zwitterionic lipids are likely to reduce the range and the strength of the attractive (van der Waals) forces between CNTs, ${ }^{30,31}$ ) allowing dipole-dipole interactions to become more prominent.

\section{Conclusions}

We have demonstrated self-assembly of lipid-coated ferromagnetic carbon nanotubes, with lengths in the 1$5 \mu \mathrm{m}$ range, into higher-order structures on the millimeter scale. These patterns, consisting of primarily end-to-end oriented individual nanotubes, have to our knowledge not previously been observed: flow-induced patterns of acidtreated and/or detergent-solubilized carbon nanotubes gave rise to a side-to-side packing of nanotubes at high CNT concentration or to isolated nanotubes at low CNT density. ${ }^{14-17)}$ Lipid bilayer functionalization and end-to-end nanotube pattern formation are relevant for the development of bioelectronic devices, potentially enabling networks of carbon nanotubes that are electrically insulated from a biological electrolyte solution to transmit highly specific binding events from bilayer-embedded receptor proteins.

\section{Acknowledgments}

This work was supported by the Biotechnology and Biological Sciences Research Council (BBSRC), the Engineering and Physical Sciences Research Council (EPSRC), the Medical Research Council (MRC) and the Ministry of Defence (MoD) through the Bionanotechnology IRC. NG acknowledges support from The Royal Society, WomenInNano and the GDRE Nano-E 2756.

1) I. Willner and B. Willner: Trends Biotechnol. 19 (2001) 222.

2) M. R. R. de Planque, G. P. Mendes, M. Zagnoni, M. E. Sandison, K. H. Fisher, R. M. Berry, A. Watts, and H. Morgan: IEE Proc.Nanobiotechnol. 153 (2006) 21.

3) E. Katz and I. Willner: ChemPhysChem 5 (2004) 1084.

4) M. Endo, T. Hayashi, Y. A. Kim, and H. Muramatsu: Jpn. J. Appl. Phys. 45 (2006) 4883.

5) H. J. Burch, S. Antoranz Contera, M. R. R. de Planque, N. Grobert, and J. F. Ryan: submitted to Adv. Mater.

6) J. Jass, T. Tjärnhage, and G. Puu: Biophys. J. 79 (2000) 3153.

7) R. P. Richter, R. Bérat, and A. R. Brisson: Langmuir 22 (2006) 3497.

8) G. Wiegand, N. Arribas-Layton, H. Hillebrandt, E. Sackmann, and P. Wagner: J. Phys. Chem. B 106 (2002) 4245.

9) B. A. Cornell, V. L. Braach-Maksvytis, L. G. King, P. D. Osman, B. Raguse, L. Wieczorek, and R. J. Pace: Nature 387 (1997) 580.

10) K. Yamamoto, S. Akita, and Y. Nakayama: Jpn. J. Appl. Phys. 35 (1996) L917.

11) X. Q. Chen, T. Saito, H. Yamada, and K. Matsushige: Appl. Phys. Lett. 78 (2001) 3714.

12) M. Senthil Kumar, S. H. Lee, T. Y. Kim, T. H. Kim, S. M. Song, J. W. Yang, K. S. Nahm, and E. K. Suh: Solid-State Electron. 47 (2003) 2075.

13) R. D. Deegan, O. Bakajin, T. F. Dupont, G. Huber, S. R. Nagel, and T. A. Witten: Nature 389 (1997) 827.

14) R. Duggal, F. Hussain, and M. Pasquali: Adv. Mater. 18 (2006) 29.

15) Q. Li, Y. T. Zhu, I. A. Kinloch, and A. H. Windle: J. Phys. Chem. B 110 (2006) 13926. 
16) H. Ko and V. V. Tsukruk: Nano Lett. 6 (2006) 1443.

17) H. Ko, S. Peleshanko, and V. V. Tsukruk: J. Phys. Chem. B 108 (2004) 4385 .

18) N. Grobert, W. K. Hsu, Y. Q. Zhu, J. P. Hare, H. W. Kroto, D. R. M. Walton, M. Terrones, H. Terrones, Ph. Redlich, M. Rühle, R. Escudero, and F. Morales: Appl. Phys. Lett. 75 (1999) 3363.

19) N. C. Toledo, M. R. R. de Planque, S. Antoranz Contera, and J. F. Ryan: in preparation for publication.

20) R. D. Deegan, O. Bakajin, T. F. Dupont, G. Huber, S. R. Nagel, and T. A. Witten: Phys. Rev. E 62 (2000) 756.

21) A. Leonhardt, M. Ritschel, D. Elefant, N. Mattern, K. Biedermann, S. Hampel, Ch. Müller, T. Gemming, and B. Büchner: J. Appl. Phys. 98 (2005) 074315.

22) S. Karmakar, S. M. Sharma, M. D. Mukadam, S. M. Yusuf, and A. K. Sood: J. Appl. Phys. 97 (2005) 054306.

$23)$ C. Prados, P. Crespo, J. M. González, A. Hernando, J. F. Marco, R. Gancedo, N. Grobert, M. Terrones, R. M. Walton, and H. W. Kroto: IEEE Trans. Magn. 37 (2001) 2117.

24) C. Prados, P. Crespo, J. M. González, A. Hernando, J. F. Marco, R.
Gancedo, N. Grobert, M. Terrones, R. M. Walton, and H. W. Kroto: Phys. Rev. B 65 (2002) 113405.

25) J. F. Marco, J. R. Gancedo, A. Hernando, P. Crespo, C. Prados, J. M. González, N. Grobert, M. Terrones, D. R. M. Walton, and H. W. Kroto: Hyperfine Interactions 139 (2002) 535.

26) C. Neto, M. Bonini, and P. Baglioni: Colloids Surf. A 269 (2005) 96.

27) L. Chitu, Y. Chushkin, S. Luby, E. Majkova, G. Leo, A. Satka, M. Giersig, and M. Hilgendorff: Appl. Surf. Sci. 252 (2006) 5559.

28) M. Fermigier and A. P. Gast: J. Colloid Interface Sci. 154 (1992) 522.

29) G. Helgesen, A. T. Skjeltorp, P. M. Mors, R. Botet, and R. Jullien: Phys. Rev. Lett. 61 (1988) 1736.

30) J. N. Israelachvili: Intermolecular and Surface Forces (Academic Press, Amsterdam, 1992) 2nd ed., p. 404.

31) J. Marra and J. Israelachvili: Biochemistry 24 (1985) 4608.

32) Y. Lyatskaya, Y. Liu, S. Tristram-Nagle, J. Katsaras, and J. F. Nagle: Phys. Rev. E 63 (2000) 011907.

33) G. A. Niklasson, A. Torebring, C. Larsson, C. G. Granqvist, and T. Farestam: Phys. Rev. Lett. 60 (1988) 1735.

34) T. A. Witten and L. M. Sander: Phys. Rev. Lett. 47 (1981) 1400. 